\title{
Visualization Based on Geographic Information in Augmented Reality
}

\author{
Kikuo Asai \\ The Open University of Japan \\ Japan
}

\section{Introduction}

This chapter is a study on the visualization techniques of geographic information in an augmented reality environment, focusing on its user interface. Augmented reality is a technology that superimposes information over a real scene, enhancing the real world with the scene-linked information. The information is overlaid onto the real scene, dynamically based on the user's viewpoint. When mixed reality is defined as a continuum of environments spread between reality and virtuality (Milgram et al., 1994), augmented reality is ranked within a certain range along the reality-virtuality continuum. An augmented reality environment has the following characteristics: 1) It combines real and virtual worlds; 2) It is interactive in real-time; and 3) Virtual objects are spatially registered in three dimensional (3D) spaces (Azuma, 1997).

In this chapter, however, the term "augmented reality" is used as a $3 \mathrm{D}$ user interface that provides a user with an interactive environment. Moreover, a desktop monitor is included as a part of the interactive tabletop environment for presenting the visual scene captured by a video camera, though a head-mounted display (HMD) has typically been used as a display device. Comparison of the user's experiences between the HMD and the licquid crystal display (LCD) monitor showed that the LCD was easier to use than the HMD under the certain conditions (Asai \& Kobayashi, 2007). It was because visual instability was caused by unstable images captured by a camera attached to the HMD. The coordinate system between workspace and visual feedback is then inconsistent in the environment using a desktop monitor, which requires user's mental transformation. In terms of interaction techniques, on the other hand, this tabletop environment gives us great merits of visual stability and tangible interaction with information or virtual objects.

There have been various visualization applications based on geographic information using the augmented reality technology. Our aim is here to introduce the basic concept of geographic information visualization using augmented reality, report an example with our lunar surface browsing tools, and show the features of the user interface in the geographic information visualization using map-based augmented reality.

First, the basic concept of geographic information visualization system is introduced by briefly describing general technology and its relevant applications of augmented reality. The geographic information visualization using augmented reality is mainly categorized into two styles: visualizing navigation information using GIS (geographic information system) in the real world and visualizing geographically embedded information using a real map on 
the desktop environment (Schmalstieg \& Reitmayr, 2006). The former is often called as mobile augmented reality, since the location-referenced information is presented by using portable devices. The various commercial-based applications of the mobile augmented reality have appeared in recent years. The latter is called as map-based augmented reality, since the static information on a printed map is enhanced with geographically embedded information superimposed onto the map. The augmented map may give users tangible interactions mediated through combination of physical and virtual objects.

Second, an example with our lunar surface browsing tools is reported. Our systems present information geographically embedded on the lunar surface map, as a user presents sticks with markers that identify the location on the map and the command she tries to control. We have developed three kinds of browsing tools for learning the exploration activities and the lunar surface investigations in the Apollo 17 mission of the National Aeronautics and Space Administration (NASA). The first browsing tool created visualization based on computer graphics. Although tangible interfaces were implemented for manipulating the geographically embedded information on the lunar surface map, the potential of augmented reality was not drawn sufficiently. The second browsing tool created visualization using three interaction techniques based on a real map. The third browsing tool was created for comparison with the above browsing tools, adopting a WIMP (window, icon, menu, pointing) interface.

Third, usability with the browsing tools is discussed on the basis of user studies. Subjective evaluation was done with preference tests in order to investigate the properties of a tangible tabletop environment created by the map-based augmented reality visualization. For the sake of the evaluation, two experiments were designed comparing the map-based augmented reality environments with a WIMP environment. The method and results of the experiments are described, and the interpretations are given taking account of the openended comments.

Finally, the above concerns are summarized, and the future direction of the map-based augmented reality visualization is described in the context of the lunar surface browsing tools.

\section{Geographic information visualization using augmented reality}

We here describe design types of geographic information visualization using augmented reality and some applications for viewing the additional information related to the geographic information. The design types are classified by environments dedicated to visualization mainly into two categories; mobile augmented reality visualization and mapbased augmented reality visualization.

\subsection{Mobile augmented reality visualization}

The mobile augmented reality provides us everywhere and anytime with a computeraugmented environment that enhances the real world with the location-based information, as shown in Figure 1. A portable device or head-mounted display (HMD) has been used as a display device. A compact video camera, embedded in the portable device or attached to the HMD, captures video images that are used for recognizing the real world and detecting physical objects in the real world. The recent downsizing technology allows the video images to be processed with a CPU in portable devices. Determining the user's position and orientation is necessary to provide the location-based information. The location data is 
obtained by GPS (global positioning system) and/or geomagnetic sensor (electrical compass) and is transferred to a server through the Internet. The server searches the relevant information based on the location data and provides the user with the relevant information. The relevant information is superimposed onto the scene images at the portable device or HMD.

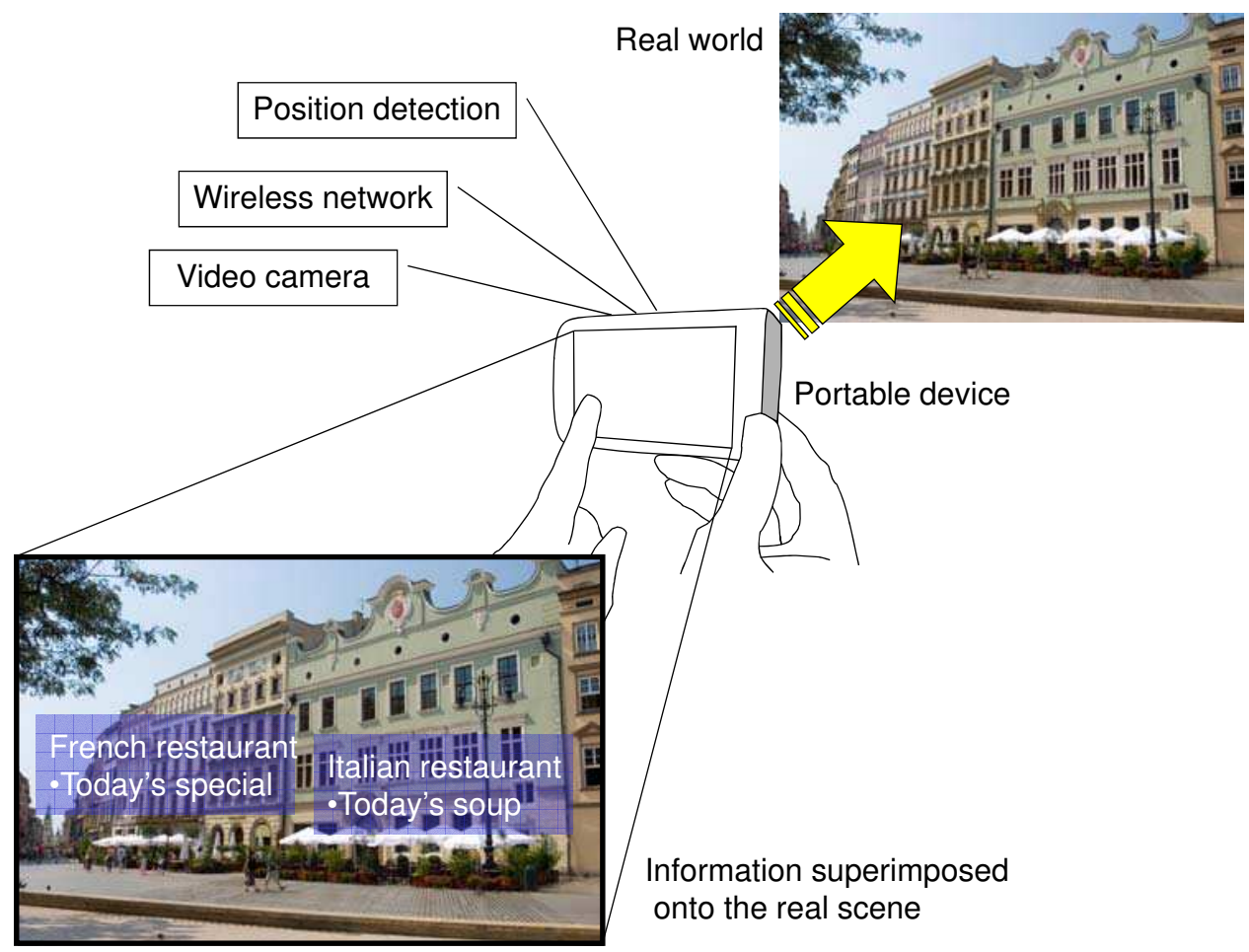

Fig. 1. Mobile augmented reality environment

There have been various systems developed as the mobile augmented reality applications. The Touring Machine was developed as a prototype system that combines augmented reality and mobile computing (Feiner et al., 1997), presenting information about the university campus at an outdoor environment. The prototype system further produced computer-aided navigation such as MARS (Hollerer et al, 1999) and Situated Documentaries (Hollerer et al., 1999). The former provided the user with an interactive multimedia presentation environment between indoors and outdoors, and the latter provided a tour guide by interactive multimedia presentations. These location-aware or location-based computing systems offer services that are related to the user's location (Beadle et al., 1997). The typical service is tour guide and navigation support applications, for example, a mobile edutainment system learning historical facts via a thrilling story, GEIST (Kretschmer et al., 2001), a personalized system touring archaeological sites, ARCHEOGUIDE (Vlahakis et al., 2003), and a tourist guide system supporting collaboration between multiple mobile users in both outdoor city areas as well as indoor areas of buildings, Signpost (Reitmayr \& Schmalstieg, 2004). 
The BAT system provided a user with an infrastructure of sensor network by using ultrasonic trackers, which offers location-based services throughout a large and populous area (Newman et al., 2001). This created a kind of ubiquitous computing environment inside a building, allowing the user to roam freely within the entire building. Since the system knows the users' locations in the building, it can give the users useful information that they would need at that time and the information about the users' locations can be shared among all the users. The Tinmith-Metro system provided a user with an authoring environment for creating 3D models interactively outdoors (Piekarski \& Thomas, 2001). The constructive solid geometry modeler was controlled by using the menu and glove user interface called Tinmith Hand, which supports interaction techniques for manipulating the 3D graphical models. The system was designed to share the 3D models among the users outdoors and indoors in real-time.

Recent portable devices have inspired commercial services of mobile augmented reality applications. The mobile technologies do not just make the handheld devices compact, but also allow the devices to embed various functions such as a video camera, positioning system, e-mail, the Internet, TV, and telephone. Key technology that makes the locationbased services successful is position detection of users, including the orientation of the handheld devices or the viewpoints of the users as well as the locations. GPS has mainly been used for determining the position outdoors. The accuracy would be improved by using magnetic sensors (electronic compass) and/or inertial sensors as well as vision-based tracking. Seamless position detection indoors is an issue addressed for providing the location-based services inside buildings. Current technologies of wireless communications used indoors include infrared communication system, wireless LAN (local area network), IMES (indoor messaging system), and QR code. The infrared communication system offers broadband network services with high security. The wireless LAN is practical because the access points of the wireless LAN currently exist are used for position detection. The positioning information services have already been provided, for example, PlaceEngine by Koozyt and Wi-Fi positioning system by Skyhook Wireless. The IMES is a kind of indoor GPS that is the most promising method from a technical point of view, because the IMES is compatible with the GPS and allows the current GPS receivers to be used with modification of the firmware. The QR code is a two dimensional bar code that is recognized with image processing of the images captured by a camera.

Integrating the above technologies produces various applications. SekaiCamera (tonchidot) is a navigation system using online data by attaching digital tags called AirTag to the real world. The AirTag is a kind of icon overlaid onto the real scene image and has the link information that provides the location information related to the place. OneShotSearch (NEC Magnus Communications) gives the user location-based information about stores, restaurants, and navigation by using a mobile phone with GPS, a magnetic sensor, and a camera. For example, names of restaurants and the direct distances are presented at an order in position closer to the user. When the user selects one of the restaurants, the direction is indicated with an arrow, navigating the user to the restaurant. Real World Perspective Mobile Phone (au One Lab) is an intuitive user interface of obtaining information about humans and objects around the user by presenting a virtual perspective view over the mobile phone. Although it uses simple graphics visualization, adaptive information to the user is presented by estimating the user's status accurately with various sensors for detecting vibration and sound as well as position. SREngine (SREngine) is a kind of search 
engine to find the spatial information from the scene images captured by a camera. The data of the scene recognition is obtained by only image processing, and the additional sensors such as GPS and wireless LAN are not necessary.

Various portable devices have had positioning system, and have been able to record the data with geographic information. Some digital camera has a function of taking photos with GPS information. The photos would be uploaded on the Internet and shared in the general public. Location-based applications with a new concept will appear in cooperation with CGM (Consumer Generated Media), creating a kind of social network.

\subsection{Map-based augmented reality visualization}

A map-based visualization system is basically a map medium on which geographic data is stored, retrieved, and presented, allowing a user to manage the data linked to location and its spatial features, as shown in Figure 2. The geographic data and the associated attributes are often mapped in one coordinate, and are layered together for the analysis. GIS has been incorporated into various applications such as scientific investigation, resource management, and development planning.

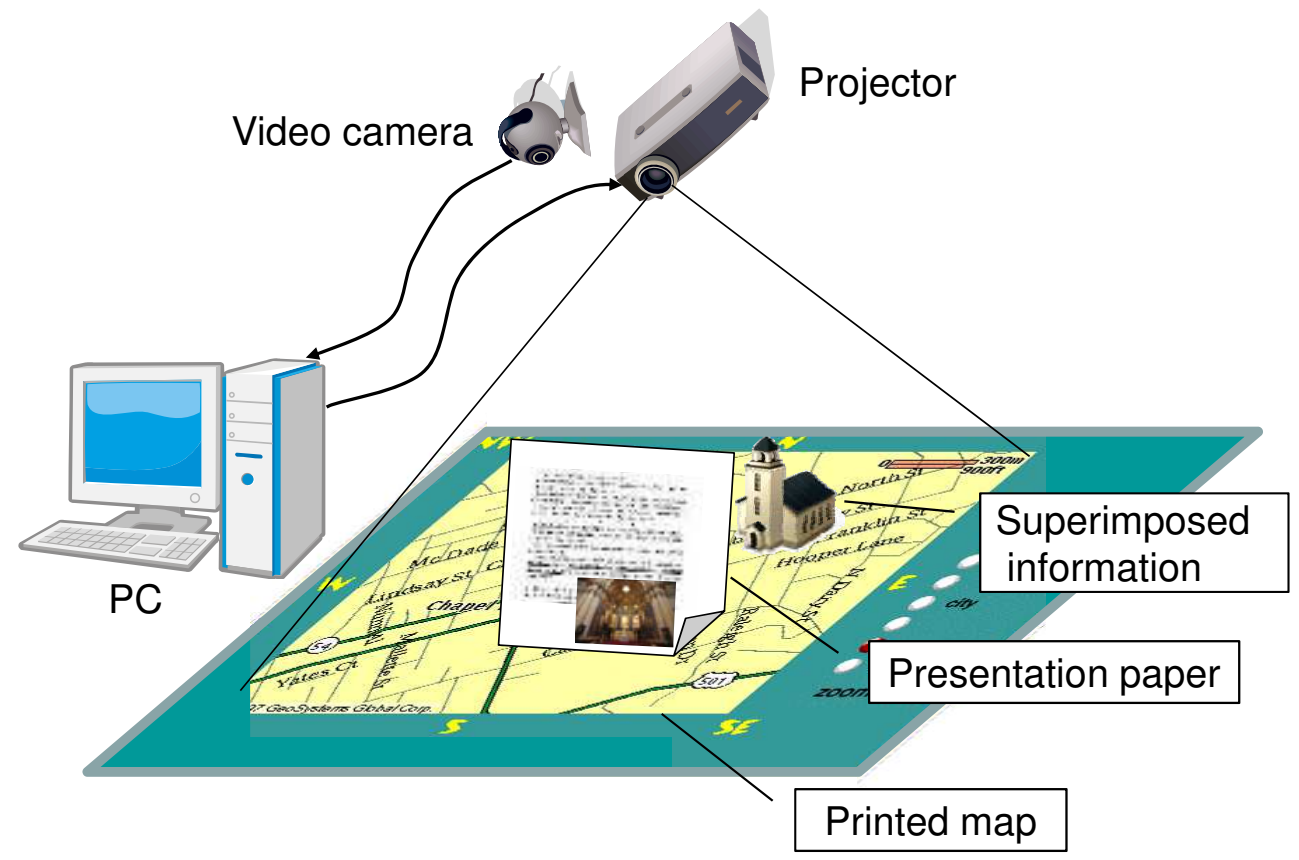

Fig. 2. Map-based augmented reality environment

Although computer-based GIS is an effective tool for visualizing geographic data, printed maps offer high resolution and simple interaction. Because printed maps display highdensity information, they support both overview browsing and detailed area investigation. Moreover, the interaction is intuitive due to the direct manipulation; maps printed on a sheet of paper can be freely moved around on a table. However, printed maps do not display information dynamically to match the user's evolving interests. 
Interaction discontinuity - the mental switch between the virtual and real worlds -creates problems in interface design when a traditional interface device such as a mouse is used for manipulating 3D objects (Poupyrev et al., 2002). Interaction discontinuity is caused by a spatial gap between the physical and virtual workspaces. One solution to this problem is a computer interface based on physical objects (Wellner, 1993). Augmented reality overlays video images of a real scene with virtual objects, creating a tangible interaction environment in which a user interacts intuitively with the virtual objects by manipulating the corresponding physical objects (Ullmer \& Ishii, 1997; Kato et al., 2000; Regenbrecht, 2001).

Augmented reality has been applied to cartography to create a user interface that bridges the gap between the computer-generated information and the everyday experiences of users (Schmalstieg \& Reitmayr, 2006). A user of map-based AR can use a printed map to flexibly access geographically embedded information by pointing at the location on the map and superimposing the information onto the map.

The application of augmented maps (Bobrich \& Otto, 2002) to cartography applications enables the visualization of 3D digital geographic data overlaid on a printed map using a video see-through HMD. A marker pointer was used as an action trigger to detect the intersections between virtual objects. The pointer is recognized as a marker in the video images of the real scene.

Augmenting maps with information projected from overhead facilitates tangible interaction in a tabletop environment (Reitmayr et al., 2005). A PDA (personal data assistant) device was used as a tangible user interface instead of a marker pointer, enabling intuitive access to information linked to locations on the printed map. The occlusion problem encountered when using multiple maps was overcome by the depth-ordering information computed at the map localization step and the overlays were improved by rendering correct occlusion with other maps. The relevant images to the location on the map are projected at the area inside a square frame.

The Illuminating Clay system (Ishii et al., 2004), a GIS interface for landscape design and analysis, uses a clay model instead of a printed map. It captures the geometry of the clay landscape model in real time with a ceiling-mounted laser scanner and projects the geometry-based information onto the clay model.

A geographic file explorer system was developed to improve retrieval and visualization of landscape data (Favetta \& Laurini, 2006). Image and video files of potential interest to a user are retrieved from a large set of geographically embedded documents, and those recorded at the place closest to the one selected on the map are presented. This provides an efficient way to quickly recall the locations of the documents.

Our browsing tools are similar in concept to these map-based visualization systems in that they provide a tangible user interface. Application of our map-based AR tools to the moon explored during the Apollo missions (NASA) is dedicated to creating a learning environment in which users can interactively browse geographically embedded information about the exploration activities and view realistic landscapes of the lunar surface from an egocentric viewpoint.

\section{Lunar surface exploration and virtual maps}

The moon is often used as learning content at science museums to enlighten laymen about astronomy and space science because the moon is the Earth's only satellite and the most familiar object in the sky. It has been extensively explored, particularly during the NASA 
Apollo missions, and much scientific data has been collected, including images of the lunar surface and audio recordings of the communication among the astronauts and between the astronauts and the ground commanders.

In this section we briefly describe the Extra-Vehicular Activities (EVA) during the Apollo 17 mission and the geographic information viewers that have existed for navigating the moon.

\subsection{Apollo 17 mission}

The Apollo 17 mission was manned-exploratory mission in a series of the project Apollo. Three astronauts, Eugene Cernan, Ronald Evans, and Harrison Schumitt have directed to the moon, and two out of the three astronauts landed on the moon.

The lunar module (LM) landed in a valley in the Taurus-Littrow region. Three separate lunar surface excursions were conducted during the stay. In the early phase of the first one, the ground-commanded television camera and high-gain antenna on the lunar roving vehicle (LRV) were installed, and video signals around the LM were sent to the Houston space center. The astronauts conducted various scientific experiments during the excursions, spending roughly 22 hours outside the LM and traversing about $35 \mathrm{~km}$ in the LRV. They collected nearly $110 \mathrm{~kg}$ of rock and soil samples from various areas of stratigraphic interest: light mantle, dark mantle, sub-floor, massifs, and the Sculptured Hills.

The navigation area for the browsing tools is shown in Figure 3. The Apollo 17 preliminary science report (NASA, 1973) contains a huge amount of data, that it is neither easy nor practical for laymen to comprehend in total. For our study, we selected several remarkable experiments and samplings that were performed during the three excursions.

In the first excursion, a deep core sample and a single core tube were taken concurrently using the Apollo lunar surface experiment package. Geological samples, including a rake sample, were collected near the rim of Steno Crater. In the second excursion, geological samples were collected at five stations (the predetermined stopping points) and at eight LRV sampling stops (intermediate points): three between the LM and station 2, one at station 2a, and four between station 3 and the LM. Geological activities in the third excursion included main sampling stops at four stations and several LRV sampling stops between the stations. The Catalog of Apollo 17 Rocks describes each of the 334 individually numbered rock samples.

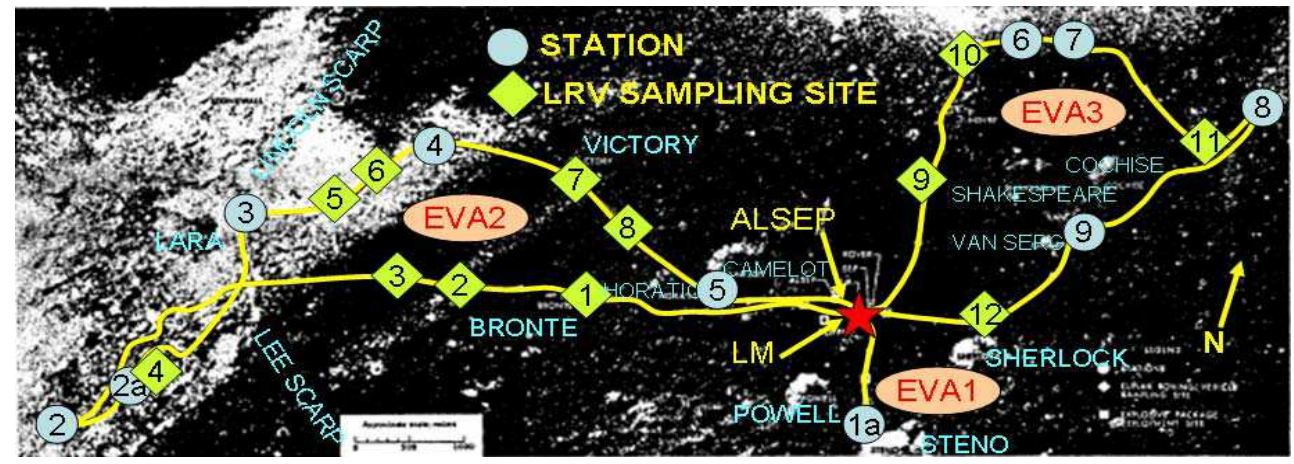

Fig. 3. Navigation area around the Apollo 17 landing site 


\subsection{Geographic information viewers for moon navigation}

There have been several navigation tools to visualize the lunar surface exploration data and to display the images of the lunar surface relief. One of these tools is World Wind (NASA), a navigation system developed by NASA to visualize geographical data using zoom-in and zoom-out functions. A person using World Wind can, for example, browse in full 3D the moon terrain mapped by the Clementine spacecraft in 1994 and view details such as craters while navigating the lunar surface. Google Moon (Google) is another navigation tool that enables viewing of each of the Apollo landing sites with a Web browser. It has a simple interface that permits the user to browse geographical data at different scales and with annotations. On July 20, 2009, the 40th anniversary of the Apollo 11 mission, Google also released Moon in Google Earth (Google). The Moon in Google Earth enables users to view high-quality images and landscapes of the lunar surface using the data obtained by spacecrafts.

Other visualization tools, such as VistaPro Renderer (VistaPro) and Kashmir 3D (Sugimoto), enable the creation of realistic 3D landscapes and viewing of geographical relief. By importing lunar geographical data into such tools, a user can generate scenes of the lunar surface that can be viewed from various viewpoints. All these viewers have been developed supposing that users would have a certain level of experiences for personal computers (PCs). At public places, however, such assumption is not often available.

If flexible learning is interactive and individual (Schmid, 1999), then a tangible interface, which tangibly presents information from the user's selected viewpoint, can improve learning flexibility. AR may enhance the learning flexibility by showing the user a miniaturized lunar surface rather than just a framed view. While conventional audiovisual learning systems efficiently present their subject matter, their lack of interaction can lead to boredom. Our lunar surface browsing tools then use the tangible AR interface for the learning flexibility, which offers seamless interaction that bridges the gap between the virtual and real worlds. Our browsing tools create a miniature environment for viewing realistic landscapes of the lunar surface, which enables users to interactively learn about exploration activities of the Apollo 17 mission.

\section{Browsing tools for learning lunar surface}

We have originally developed a lunar surface navigation system at the immersive projection display environment (Kobayashi \& Asai, 2003) that gave the user a wide field of view and a strong feeling of "being there." The system was quite large, however, limiting its use outside the laboratory. We then developed two browsing tools as a PC-based learning environment, which would be used for an exhibition at science museums. With these tools users can browse geographically embedded information in the lunar surface areas related to the Apollo 17 mission (NASA). The browsing tools have visualization functions in order to facilitate comprehension of the geographical features around the Apollo 17 landing site.

\subsection{Computer graphics based visualization}

The first version of the lunar surface browsing tool has rendered the lunar surface with computer graphics instead of a paper map (Asai et al., 2006). It kept visual compatibility of the geographic position, because of no registration problem of virtual objects onto the real scene. The browsing tool has provided a tangible user interface by using marker sticks. The 
user manipulates a virtual space with the sticks whose markers work as control commands when presented in the scene and captured by a camera. Figure 4 (a) shows an overview of the lunar surface browsing tool. Figure 4 (b) shows the markers used for controlling the virtual space and identifying the location where the lunar surface model should be superimposed in the captured scene.

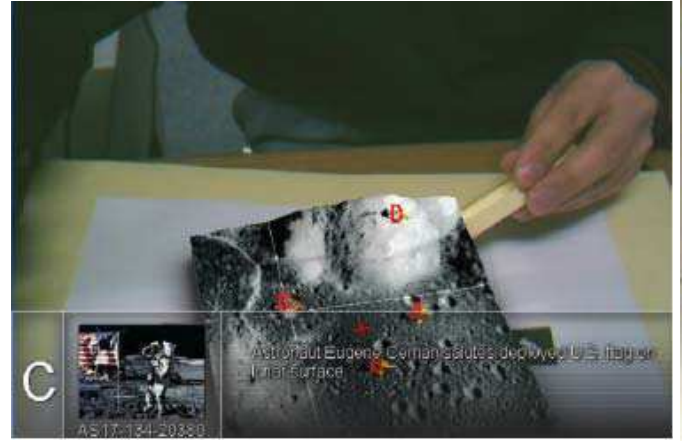

(a)Lunar surface navigation

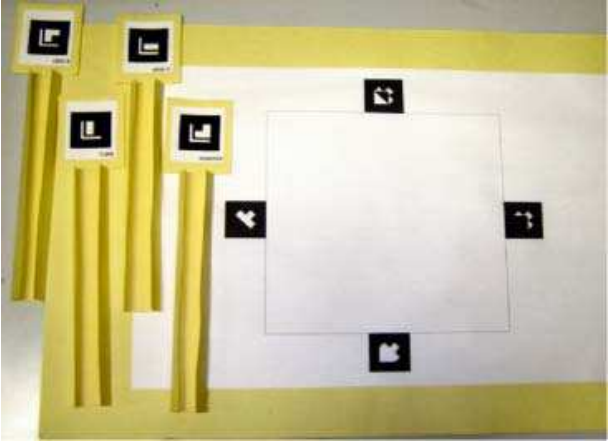

(b) Control command and base markers

Fig. 4. Overview of the first lunar surface browsing tool

\subsubsection{Visualization functions}

Markers are assigned for the following navigation functions.

1. Pointer: By pointing this marker (orange cone in Fig. 5 (a)) at a certain location on the lunar surface, the user can shift the area in view and present the relevant location information.

2. Area shift: Pointing with this maker allows the user to navigate to the indicated location. Pressing the space bar on the keyboard centers the pointing place on the view.

3. Information presentation: Users point at a label and information pops up on a transparent panel (Fig. 4 (a)). The information-generally images and text or sounds -is relevant to the location on the lunar surface.

4. Rotation: The rotation marker changes the viewpoint by rotating the entire area of the lunar surface in view (Fig. 5 (b)).

5. Zoom: Users change the scale of the lunar surface map-zooming in and out-by rotating the mouse wheel (Fig. 5 (c)).

6. Grid: The grid marker overlays a grid on the lunar surface and presents information about the scale of the area along the edge of the image (Fig. 5 (d)).

7. Altitude: Inserting the altitude marker into the video image brings up a color contour for identifying valleys, scarps, and 3D geometrical relief (Fig. 5 (e)).

8. Rover's path: Inserting the path marker into the image area reveals the path taken by the rover (Fig. 5 (f)). The viewing area can also be automatically shifted along the rover's path.

The multimedia content embedded along the path taken by the LRV is revealed by manipulating the navigation system, allowing us to learn about the exploratory activities. The audiovisual materials - photos and sounds recorded during the activities - realistically describe the situation on the lunar surface. The voice communication between the astronauts and the space center is particularly effective in evoking a realistic sensation of presence. The shading on the lunar surface model also helps to create a realistic landscape. 


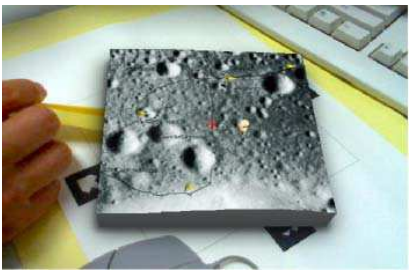

(a) Pointer

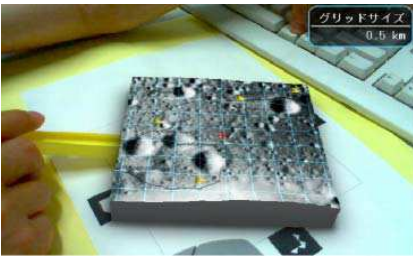

(d) Grid

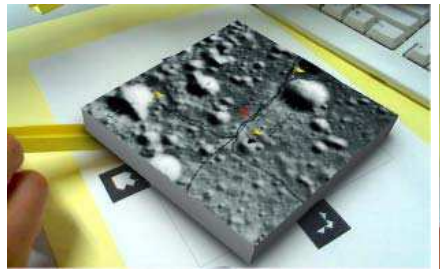

(b) Rotation

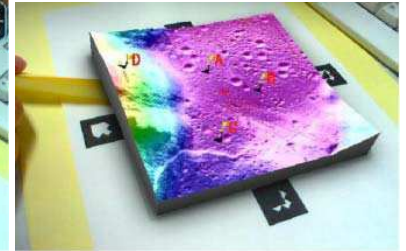

(e) Altitude

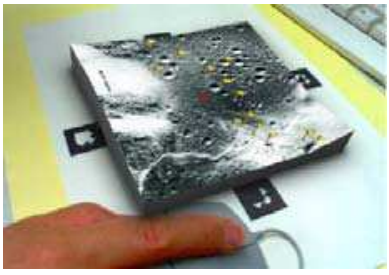

(c) Zoom

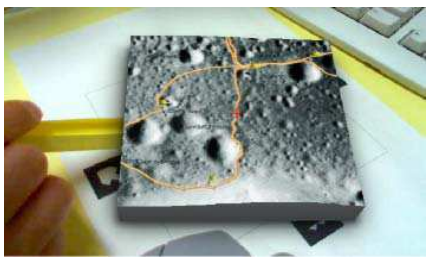

(f) Path of the LRV

Fig. 5. Visualization functions of the first version

\subsubsection{Implementation}

This system uses a parameter settings file to designate the text and audiovisual files presented as multimedia content. Topographic data of the Taurus-Littrow region was used for the navigation map. All of the data, text, images, and audio are linked to locations where the astronauts conducted scientific activities. Voice communication between the astronauts can be heard by touching the pointer to a voice label. The data were obtained from NASA's web site for the Apollo missions (NASA).

The PC was equipped with a $2.6 \mathrm{GHz}$ Pentium IV, $512 \mathrm{MB}$ of memory, and an nVidia GeForce FX5700 graphics card with 128 MB of video RAM. Video images were captured with a $640 \times 480$-pixel web camera. ARToolkit (HITLAB) was used to process images, detect the square markers, and track the positions and orientations of the markers.

\subsection{Real map based visualization}

The second version of the lunar surface browsing tool has used a real map as a medium presenting the lunar surface instead of rendering computer graphics (Asai et al., 2008). A tangible tabletop environment was provided by interacting with a printed map of the lunar surface and marker sticks of the control commands. A camera mounted above the map captures the video images that are processed to recognize the patterns of the markers. Tracking the marker's location enables the user to view information (photos, texts, and sounds) associated with the location on the map. Figure 6 shows an overview of the second lunar surface browsing tool, presenting the geographically embedded information (Fig. 6 (a)) and the tabletop environment with the lunar surface map and markers (Fig. 6 (b)).

The geographically embedded information in Fig. 6 (a) includes an image of the LRV and annotations about the geographic features at station 5 that are revealed by pointing at the label on the map. The annotations are read out as narration at that time, so that people can understand the difficult words. If a voice label on the map is selected, the system plays a recording of the voice communication during the exploration in the Apollo 17 mission. The sort of labels is distinguished with color. 
The browsing tool used three interaction techniques; (1) A pointer is used for selecting points of interest on the map by zooming and focusing, enabling reliable selection of points from among many points, (2) A slider is used for designating a range of distance along a path on the map by adjusting the length between two markers, enabling variable viewpoint control, and (3) A local view is simulated at a designated point, enabling dynamic visualization from an egocentric viewpoint.

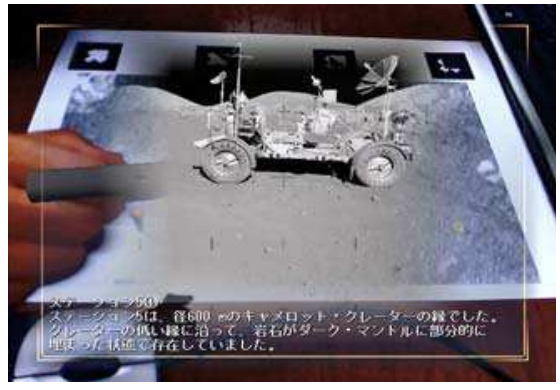

(a) Ggeographically embedded information

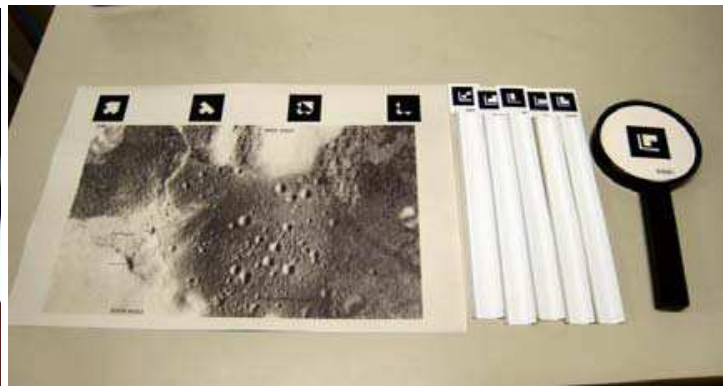

(b) Lunar surface map and markers

Fig. 6. Overview of the second lunar surface browsing tool

\subsubsection{Visualization functions}

The browsing tool in the second version has several visualization functions controlled with the control command markers.

1. Lenses Two lenses are used for accessing the zoom-in views of the lunar surface relief. The user pans one of them over the lunar surface map, reducing and increasing the zoom-in rate by changing the relative size of the lens marker in the video images (Fig. 7 (a)). The other is used as a pointer to retrieve the geographically embedded information by selecting a point of interest on the lunar surface map (Fig. 7 (b)).

2. EVA data The geographical data around the EVA are overlaid on the lunar surface map. The data include the LRV's route, the stations (Fig. 7 (c)) where the LRV stopped, and the craters near the route. The user can find their positions, names and sizes.

3. Contour Transparent images of the elevation are overlaid on the lunar surface map with contour lines (Fig. 7 (d)) or with a contour color map. The user can find the massifs northeast and southwest.

4. Grid Blue grids are overlaid on the lunar surface map for presenting the scale of the EVA area (Fig. 7 (e)). The user can understand the rough extent. The scale size of each grid is set using a parameter file.

5. LRV track A miniature of the LRV is presented that moves along the actual LRV's route on the lunar surface map (Fig. 7 (f)). The LRV miniature is quite small on the map. Then, a lens enables the user to see it on a larger scale.

6. LRV view The view of the landscape from the LRV's viewpoint is presented at another window in the video images (Fig. 7 (f)). As the LRV moves along the track, the landscapes are animated corresponding to the viewpoints along the route. The window of the LRV view is presented at the size of the LRV view marker.

7. Slider The position of the LRV on the map changes with distance between the slider markers (Fig. 7 (f)). The LRV view enables the user to view the landscape animations at the viewpoints along the route designated with the slider. 


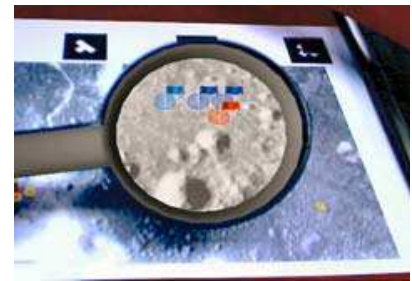

(a) Lens

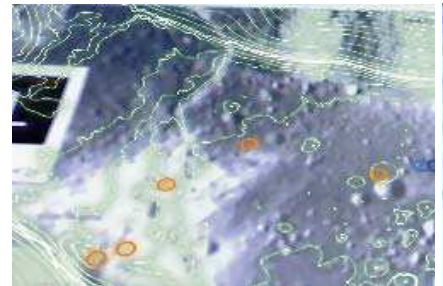

(d) Contour lines

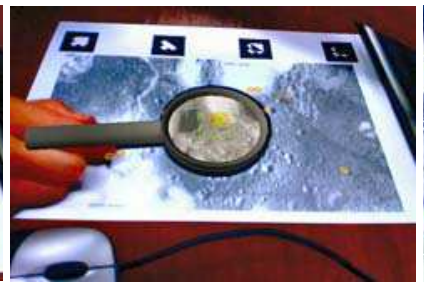

(b) Pointer

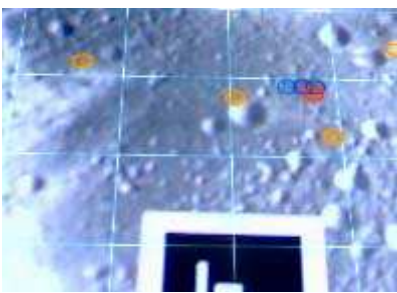

(e) Grid pattern

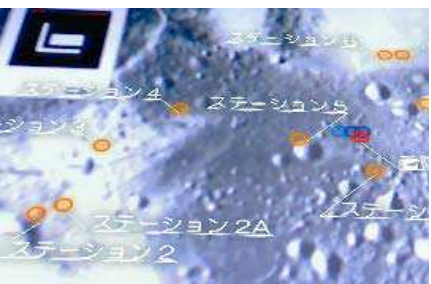

(c) Name of the stations

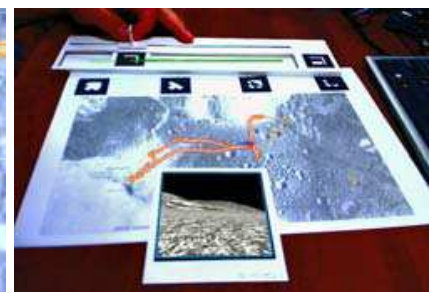

(f) Local view with the slider

Fig. 7. Visualization functions of the second version

\subsubsection{Implementation}

This system uses a parameter setting file to designate the text and audiovisual files as the geographically embedded information. A photograph of the Taurus-Littrow region was used as a printed map, and topographic data were used to make the contour lines and contour map, which were obtained by the satellite measurements. All of the documents (including annotations, images, and sounds) are linked to the corresponding locations. The data were obtained from NASA's Web sites for the Apollo missions (NASA).

The software for the browsing tool was installed on the PC equipped with a 2.6-GHz Pentium IV CPU, 512 MB of memory, and an nVidia GeForce FX5700 graphics card with 128 MB of video RAM. Video images were captured with a $640 \times 480$-pixel web camera. ARToolKit (HITLAB) was used as the image processing library for capturing the video images, detecting the square markers in the images, tracking the positions and orientations of the markers, and recognizing the patterns inside the markers.

\subsection{WIMP based visualization}

GeoMovie Creator (GMC) was originally designed for outdoor education (Hashimoto, 2004). Users can navigate the $3 \mathrm{D}$ area on a topographical map that is generated from topographical data from the Geographical Survey Institute (GSI) or from an aerial photo mapped to coordinates from the altitude data. The user can view the geographical landscape and embed additional information, related to the locations in which they are interested, with a WIMP (window, icon, menu, pointing) interface. Personal data files such as photos and videos that contain geographical GPS data are linked on the map. In these ways, students and teachers can create geographic 3D content.

We imported the lunar surface map data and relevant information into GMC, and used the tool as a WIMP interface. Figures 8 (a) and (b) show the graphical user interface and the information presentation, respectively. A red label indicates a location with embedded 
information. When the label is double-clicked, the information appears in another window. The small 2D map at the bottom right in Figure 8 (a) helps users to locate themselves on the map. The viewpoint is controlled mainly by using the arrow keys on a keyboard in combination with the shift key.

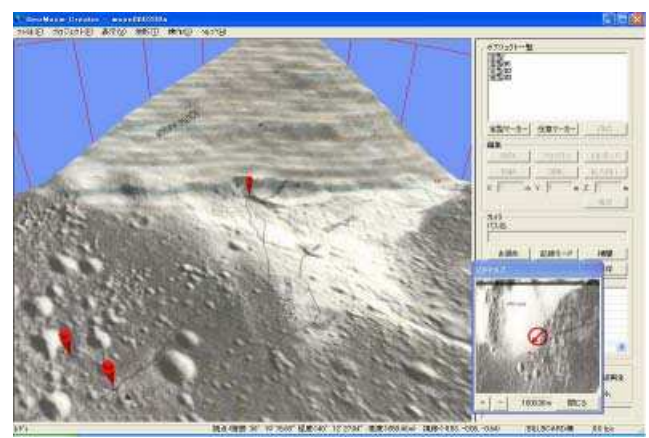

(a) Graphical user interface

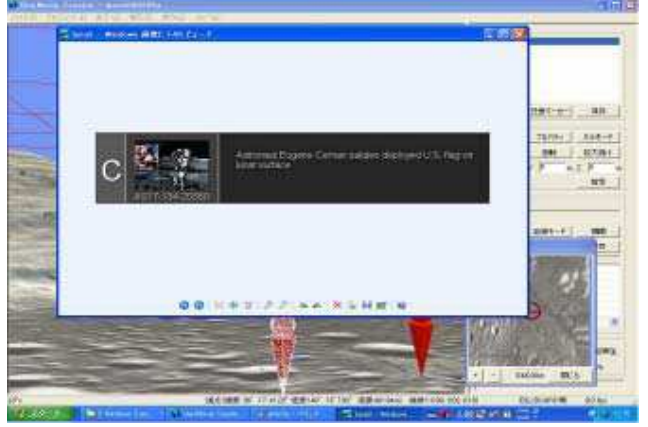

(b) Information presentation

Fig. 8. Overview of the GeoMovie Creator (GMC)

\section{User study}

We conducted user studies to compare the properties of navigation in a tangible tabletop environment with those of navigation in a WIMP environment. Sujective evaluation was done with preference tests in Experiment I and II.

\subsection{Experiment I}

We evaluated the tabletop environment in comparison to a WIMP environment in order to investigate the usability of the first lunar surface browsing tool. The computer graphics based visualization tool that we developed was used as the tabletop environment. The geographic information viewer, GMC (Hashimoto, 2004), described in the previous section was used as the WIMP environment. Figures 9 (a) and (b) show the aspects of navigation manipulation at the tabletop and WIMP environments, respectively.

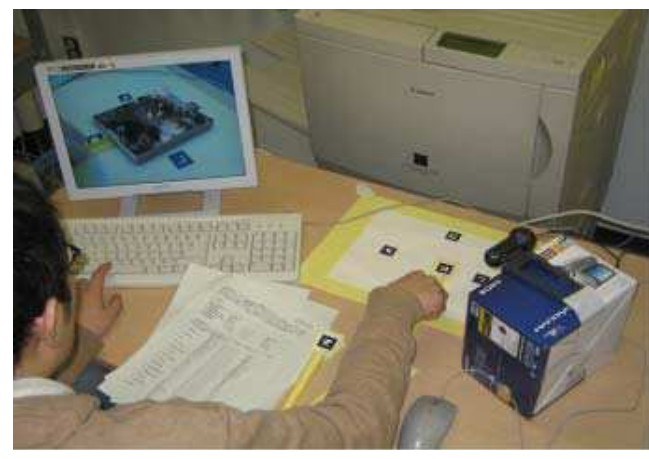

(a) Tabletop environment

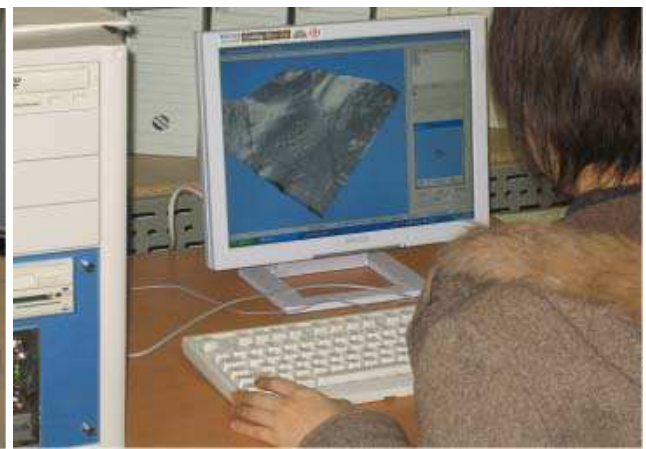

(b) WIMP environment

Fig. 9. Overview of Experiment I 


\subsubsection{Method}

Thirty undergraduate and graduate students participated in the experiment. They were asked to navigate the virtual environments of the lunar surface and retrieve information about the exploration activities of the Apollo 17 mission. The participants evaluated the usability and manipulability of the tabletop and WIMP environments. Afterwards, each participant was asked to rate the preference for the statements listed in Table 1. Open-ended comments were subsequently elicited.

\begin{tabular}{c|l} 
No. & \multicolumn{1}{|c}{ Question } \\
\hline 1 & The graphics images were stable. \\
\hline 2 & The navigation was easy. \\
\hline 3 & The relevant information was obtained easily. \\
\hline 4 & It is easy to learn how to use the system. \\
\hline 5 & Manipulating it was enjoyable. \\
\hline 6 & The manipulation was intuitive. \\
\hline 7 & Using the system for a long-time is tolerable.
\end{tabular}

Table 1. Statements of the preference test in Experiment I

\subsubsection{Results}

Figure 10 shows the results of the preference test in the Experiment I. The bar indicates the average of the five-step rating given by the participants, and the error bar indicates the standard deviation. The white and black bars correspond to the tabletop and WIMP environments, respectively. A one-way ANOVA (analysis of variance) was performed to identify the effect of the environment for each item of the preference test. A statistically significant difference was observed for Item 1 , and a tendency toward significant effects was found for Items 2 and 6. No significant difference was found for the other items.

According to Item 1, the workspace of the tabletop environment was considered inferior to that of the WIMP environment, because the instability was caused by the graphics registration based on the location of the marker in the video image. Unfortunately, the participants tended to rate navigation in the tabletop environment as inferior to that in the WIMP environment, which was more familiar to the participants. However, Item 6 showed that the participants thought navigation manipulation to be more intuitive in the tabletop environment than in the WIMP, which suggests that the augmented reality based system may achieve a better score for navigation once its stability is improved through spatial registration of the lunar surface map with the detected markers. These results were consistent with the open-ended comments. Many participants mentioned that the tabletop environment was enjoyable while the WIMP environment was accurate and stable during viewpoint transitions.

The tabletop environment had some drawbacks. Navigation was mainly accomplished by manipulating control-command markers, but changing the viewpoint needed to manipulate both markers and the keyboard. Moving to another location required pointing the marker at the desired location and pressing the space bar on the keyboard. This combination seemed intuitive, but was not suitable for controlling the viewpoint. Moreover, the camera that captured the real scene was fixed, which invalidated the advantage of augmented reality 


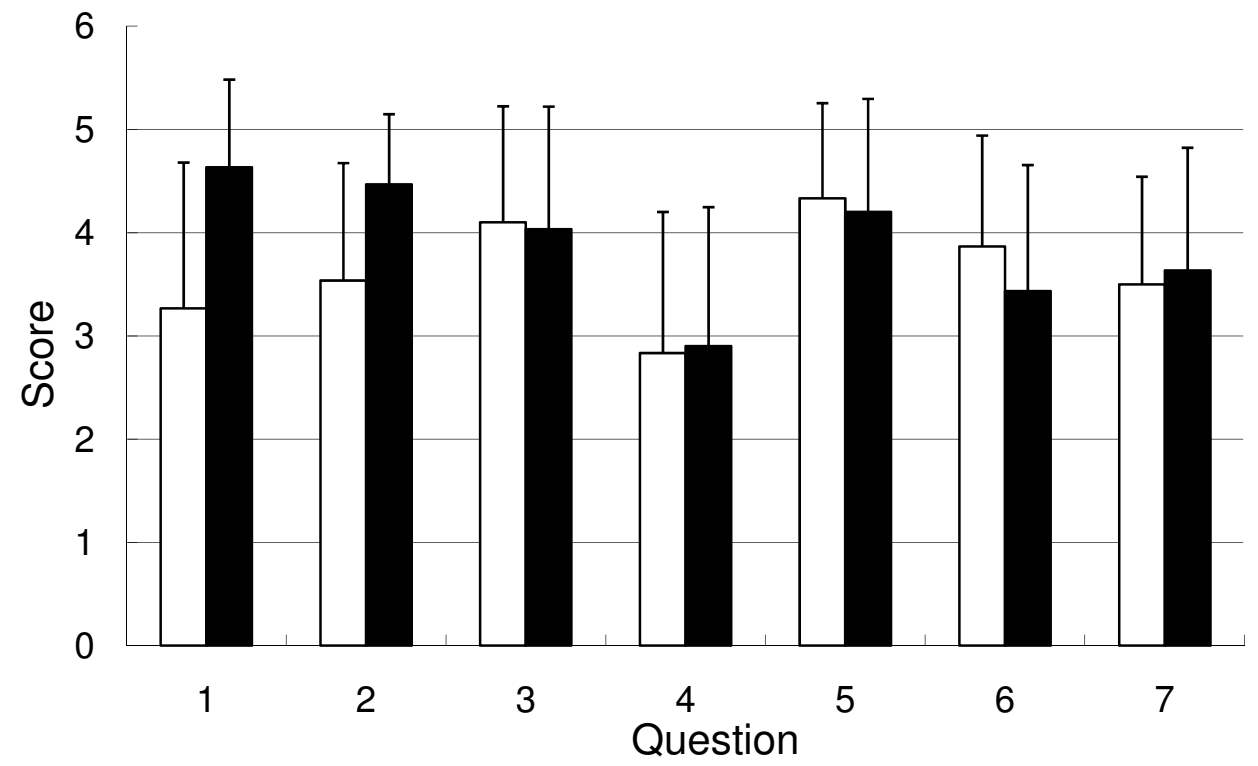

Fig. 10. Results of Experiment I (white: tabletop, black: WIMP)

interfaces in matching the virtual viewpoint to that of the user. Some participants suggested that the augmented reality based environment was exocentric, and the WIMP based one was rather egocentric, which was the opposite of what we expected.

\subsection{Experiment II}

We evaluated the map-based augmented reality environment in comparison to a WIMP environment in order to investigate the usability of the second lunar surface browsing tool (Asai et al., 2008). The real map based visualization system that we developed was used as

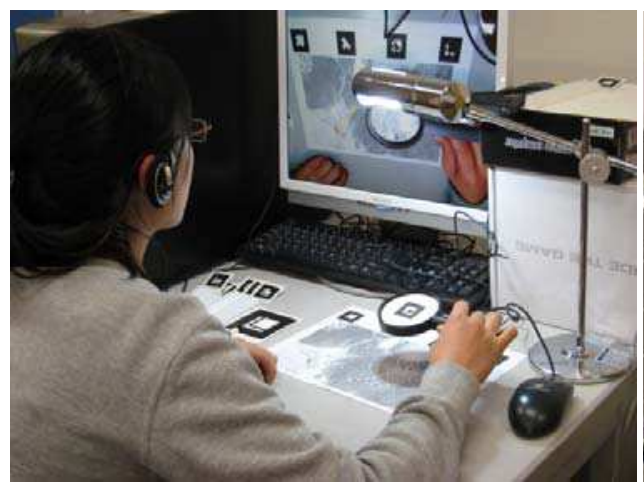

(a) Map-based AR environment

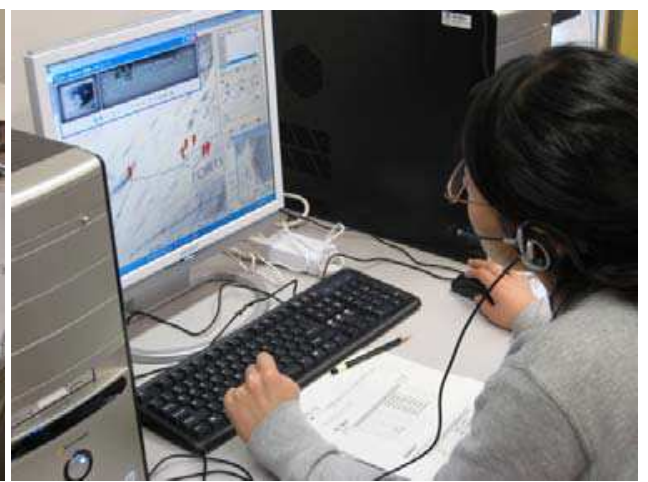

(b) WIMP environment

Fig. 11. Overview of Experiment II 
the map-based augmented reality environment. The geographic information viewer, GMC (Hashimoto, 2004), was used as the WIMP environment. Figure 11 shows examples of the tool utilization in Experiment II, indicating (a) map-based AR and (b) WIMP environments, respectively.

\subsubsection{Method}

Nineteen undergraduate and graduate students, who were not astronomy majors, participated in the Experiment II. They were instructed to use the system to become familiar with the lunar surface area explored by the Apollo 17 mission and to subsequently evaluate the operation of the browsing tool by answering eight questions in the preference test (Table 2) using a five-point scale.

They were not given any particular task, but they were given as much time as they wanted to use the system. We used an LCD monitor for the display, and the camera was placed next to the monitor on a table.

\begin{tabular}{c|l} 
No. & \multicolumn{1}{|c}{ Question } \\
\hline 1 & Was the system easy to use? \\
\hline 2 & Did the system give you an immersive feeling? \\
\hline 3 & Were you able to look over the entire area explored by the Apollo 17 mission? \\
\hline 4 & Was using the system for a long time tolerable? \\
\hline 5 & Do you think children would be able to use the system? \\
\hline 7 & Do you think people in general would be able to use the system? \\
\hline 8 & Was manipulating the system enjoyable? \\
\hline
\end{tabular}

Table 2. Questions of the preference test in Experiment II

\subsubsection{Results}

The results of the preference test are plotted in Figure 12. The black and white bars indicate the average scores for the map-based AR and WIMP environments, respectively. The error bars represent the standard deviation. Since the scores for all the questions for both environments were higher than 3 , it suggests that both should be suitable for learning about the lunar surface.

A dependent t-test was performed to identify the effect of the environment for each question. A statistically significant difference was found only for questions $1(t(18)=-3.77$, $p$ $<0.05)$ and $5(\mathrm{t}(18)=2.28, \mathrm{p}<0.05)$.

The WIMP environment was considered to be easier to use (Q1) probably because it was more familiar. Four participants pointed out in their comments that experience is needed in order to become adept at using the marker sticks for the map-based AR environment. However, as shown by the response to Q5, the map-based AR environment was considered to be better for children. Six open-ended comments pointed out that children would probably enjoy manipulating the marker sticks as well as exploring the lunar surface. The comments were consistent with the score result in Q7, which had tendency that the mapbased AR environment was more enjoyable than the WIMP environment. 


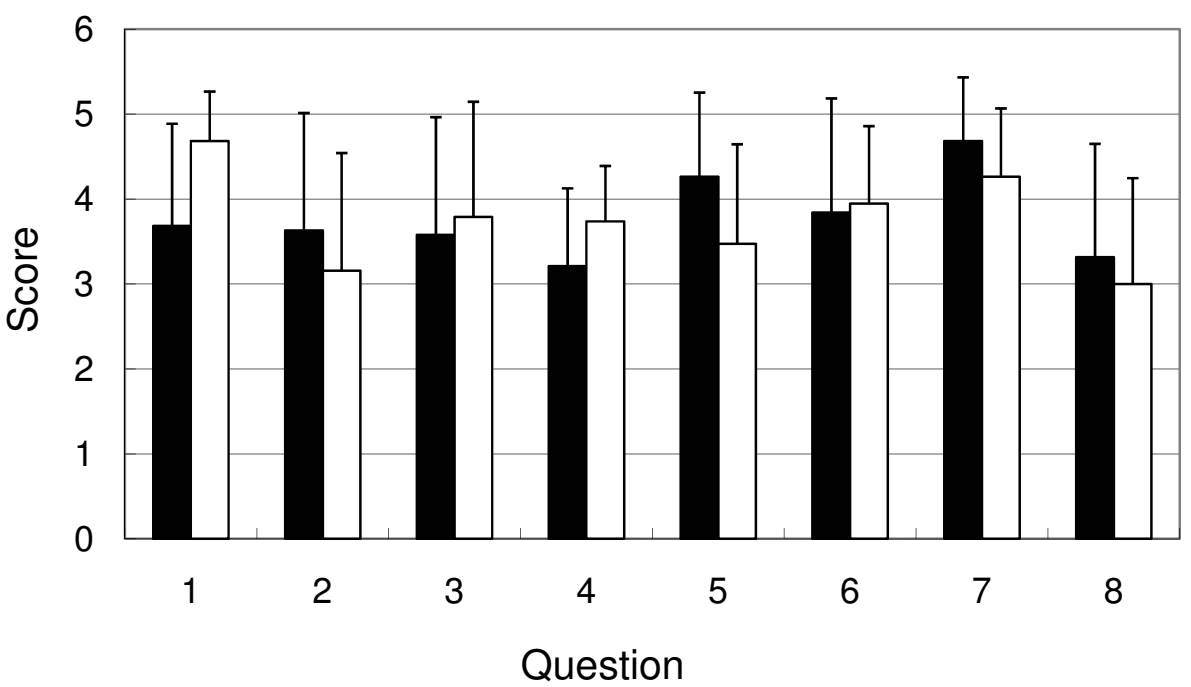

Fig. 12. Results of Experiment II (black: map-based AR, white: WIMP)

The open-ended comments from the participants give us useful suggestions to clear the characteristics of the map-based AR environment. In the positive opinions, five participants commented on the intuitive interaction using the marker sticks, and three commented on a feeling of presence created by the manipulation of the sticks and map. These were interpreted as the benefits of the tangible user interface. In the negative opinions, two participants stated that there were too many marker sticks, and nine stated that the pointing to geographically embedded information was sometimes troublesome due to the density of points within the small map at which the information embedded. Three commented on occasional confusion about the locations of the information on the map. From these comments it would be necessary to make the lens pointing function smoother.

\section{Conclusion and future direction}

We have developed lunar surface browsing tools using a tangible tabletop environment for visualizing geographically embedded information. They enable users to explore the Apollo 17 landing site, to follow the paths taken by the astronauts, and to study the extra-vehicular activity and scientific experiments. Map-based AR enhances the interactivity in geographic visualization, and these browsing tools have capabilities to provide a learning environment that improves the effectiveness of multimedia presentations.

We performed user studies in order to investigate usability of the lunar surface browsing tools, comparing them to a WIMP-based interface. The results showed that the map-based $\mathrm{AR}$ interface was enjoyable to use but unstable in the image presentations. The results also suggested that the map-based AR interface would be suitable for learning about the lunar surface and better for children than the WIMP-based interface. 
Although the egocentric landscapes were presented in computer graphics in the map-based AR browsing tool, the whole lunar surface was seen flat because of the physical limitation of the map presentation. We expected the evaluation result that the lunar surface presentation with the fine texture would highly contribute to a feeling of presence or immersion. However, this did not work well for presenting the lunar surface relief. The 3D relief was not understandable even by overlaying the contour lines or contour map over the printed map. We are investigating ways to effectively visualize the superimposition of the $3 \mathrm{D}$ relief onto the actual map.

An LCD monitor was used as a display device for presenting a real scene of the lunar surface map, and the camera was placed next to the monitor on a table. One of the advantages of augmented reality is, however, viewpoint-based interaction in which virtual objects are presented to match the user's view. This requires attaching a camera to the user's head, which makes the scene view unstable due to the head movement. This issue needs to be addressed for fully turning a map-based AR interface to advantage. Future work also includes a user study to investigate the usability of the browsing tools in practical situations, such as science museum exhibits.

\section{References}

Asai, K. \& Kobayashi, H. (2007). User study of augmented reality display interfaces for a lunar surface navigation system, Proceedings of Human-Computer Interaction International, 796-800.

Asai, K.; Kobayashi, H., Kondo, T. \& Sugimoto, Y. (2008). Subjective evaluation of a lunar surface browsing tool using map-based AR, Proceedings of International Conference on Computers in Education, pp.749-753.

Asai, K.; Kondo, T., Kobayashi, H. \& Mizuki, A. (2006). Tangible navigation system for learning the lunar surface, Proceedings of International Conference on Interactive Computer Aided Learning (CD-ROM).

Asai, K.; Kondo, T., Kobayashi, H. \& Mizuki, A. (2008). A geographic surface browsing tool using map-based augmented reality, Proceedings of International Conference Visualization, pp.93-98.

Azuma, R. (1997). A survey of augmented reality, Presence: Teleoperators and Virtual Environments, vol.6, pp.355-385.

au One Lab, Real World Perspective Mobile Phone, http:/ / kazasu.mobi/

Beadle, H. W. P.; Harper, B., Maguire, G. Q. J. \& Judge, J. (1997). Location Aware Mobile Computing, Proceedings of IEEE/IEE International Conference on Telecommunications, pp. 1319-1324.

Bobrich, J. \& Otto, S. (2002). Augmented maps, Proceedings of Symposium on Geospatial Theory, Processing, and Applications, in IAPRS, vol. 34, part 4

Favetta, F. \& Laurini, R. (2006). Visualization and exploration of landscape architecture project information, Proceedings International Conference on Distributed Multimedia Systems, pp. 153-156

Feiner, S.; MacIntyre, B., Hollerer, T. \& Webster, A. (1997). A touring machine: prototyping 3D mobile augmented reality systems for exploring the urban environment, Proceedings of Internationa Symposium on Wearable Computing, pp.74-81.

Geographical Survey Institute, Global Map, http://www.gsi.go.jp/ENGLISH/index.html/ Google, Google Moon, http://www.google.com/moon/ 
Google, Google Earth, http:/ / earth.google.com/

Hashimoto, M. (2004). GeoMovie Creator (GMC): authoring tool for creating 3D educational contents, Proceedings of Information processing Society of Japan (Japanese)

HITLAB, University of Washington, ARToolKit, http://www.hitl.washington.edu/artoolkit/

Hollerer, T; Feiner, S., and Pavlik, J. (1999). Situated documentaries: embedding multimedia presentations in the real world, Proceedings of International Symposium on Wearable Computing, pp.79-86.

Hollerer, T.; Feiner, S., Terauchi, T., Rashid, G., and Hallaway, D. (1999). Exporing MARS: developing indoor and outdoor user interfaces to a mobile augmented reality system, Computers \& Graphics, vol.23, pp.779-785.

Ishii, H.; Ratti, C., Piper, B., Wang, Y., Biderman, A. \& Ben-Joseph, E. (2004). Bringing clay and sand into digital design - continuous tangible user interfaces, BT Technology Journal, vol. 22, no. 4, pp. 287-299.

Kato, H.; Billinghurst, M. Poupyrev, I., Imamoto, K. \& Tachibana, K. (2000). Virtual object manipulation on a table-top AR environment, Proceedings of International Symposium on Augmented Reality, pp. 111-119.

Kobayashi, H. \& Asai, K. (2003). Moon-surface navigation system using immersive projection display, Proceedings of International Conference on Computers in Education, pp. 1120-1123

Kretschmer, U.; Coors, V., Spierling, U., Grasbon, D., Schneider, K., Rojas, I. \& Malaka, R. (2001). Meeting the spirit of history, Proceedings of the 2001 conference on Virtual reality, archeology, and cultural heritage, pp. 141-152.

Milgram, P.; Takemura, H., Utsumi, A. \& Kishino, F. (1994). Augmented reality: a class of displays on the reality-virtuality continuum, Proceedings of Telemanipulator and Telepresence Technplogies, SPIE, pp.282-292.

NASA. (1973). Apollo 17 Preliminary Science Report, SP-330

NASA, Apollo 17 mission, http://science.ksc.nasa.gov/history/apollo/apollo-17/apollo17.html

NASA, World Wind and the Moon, http://worldwind.arc.nasa.gov/moon.html

NEC Magnus Communications, Pointing Access Platform, http://necmagnus.jp/GViD/

Newman, J.; Ingram, D. \& Hopper, A. (2001). Augmented reality in a wide area sentient environment, Proceedings of IEEE and ACM International Symposium on Augmented Reality, pp.77-86.

Piekarski, W. \& Thomas, B. H. (2001). Tinmith-Metro: new outdoor techniques for creating city models with an augmented reality wearable computer, Proceedings of IEEE International Symposium on Wearable Computing, pp.31-38.

Poupyrev, I.; Tan, D. S., Billinghurst, M., Kato, H., Regenbrecht, H. \& Tetsutani, T. (2002). Developing a generic augmented reality interface, IEEE Computer, vol. 35, pp. 4450.

Regenbrecht, H.; Baratoff, G. \& Wagner, M. T. (2001). A tangible AR desktop environment, Computer \& Graphics, vol. 25, pp. 755-763

Reitmayr, G.; Eade, E. \& Drummond, T. (2005). Localisation and interaction for augmented maps, Proceedings of International Symposium on Mixed and Augmented Reality, pp. 120-129. 
Reitmayr, G. \& Schmalstieg, D. (2004). Collaborative augmented reality for outdoor navigation and information browsing, Proceedings of Symposium on Location Based Services and TeleCartography, pp.53-62.

Schmalstieg, D. \& Reitmayr, G. (2006). Augmented reality as a medium for cartography, Multimedia Cartography, 2nd ed. W. Cartwright, M. Peterson, G. Gartner, SpringerVerlag, pp. 267-281.

Schmid, C. (1999). Simulation and virtual reality for education on the Web, Proceedings of EUROMEDIA'99, pp.181-188.

SREngine, http://www.srengine.com/

Sugimoto, T., Kashmir 3D, http://www.kashmir3d.com/index-e.html

tonchidot, Sekai Camera, http:/ / sekaicamera.com/

VistaPro Renderer, http:/ /www. Monkeybyte.com/

Vlahakis, V.; Ioannidis, N., \& Karigiannis, J. (2003). ARCHEOGUIDE: challenges and solutions of a personalized augmented-reality guide for archaeological sites, IEEE Computer Graphics \& Applications, vol.22, pp.52-60.

Ullmer, B. \& Ishii, H. (1997). The metaDESK: models and prototypes for tangible user interfaces, Proceedings of ACM Symposium on User Interface Software and Technology, pp. 223-232

Wellner, P. (1993). Interaction with paper on the digital desk, Communications of the ACM, vol.36, pp.87-96. 


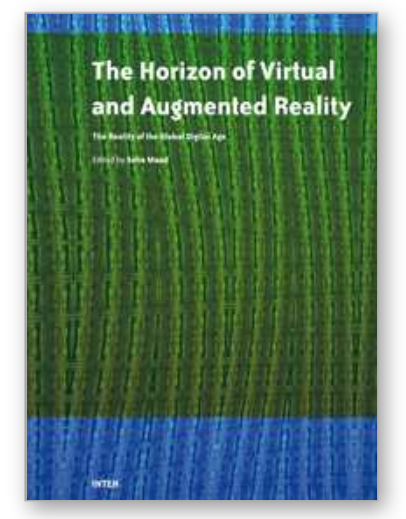

\author{
Augmented Reality \\ Edited by Soha Maad
}

ISBN 978-953-7619-69-5

Hard cover, 230 pages

Publisher InTech

Published online 01, January, 2010

Published in print edition January, 2010

Virtual Reality (VR) and Augmented Reality (AR) tools and techniques supply virtual environments that have key characteristics in common with our physical environment. Viewing and interacting with 3D objects is closer to reality than abstract mathematical and 2D approaches. Augmented Reality (AR) technology, a more expansive form of VR is emerging as a cutting-edge technology that integrates images of virtual objects into a real world. In that respect Virtual and Augmented reality can potentially serve two objectives: reflecting realism through a closer correspondence with real experience, and extending the power of computer-based technology to better reflect abstract experience. With the growing amount of digital data that can be stored and accessed there is a rising need to harness this data and transform it into an engine capable of developing our view and perception of the world and of boosting the economic activity across domain verticals. Graphs, pie charts and spreadsheet are not anymore the unique medium to convey the world. Advanced interactive patterns of visualization and representations are emerging as a viable alternative with the latest advances in emerging technologies such as AR and VR. And the potential and rewards are tremendous. This book discusses the opportunities and challenges facing the development of this technology.

\title{
How to reference
}

In order to correctly reference this scholarly work, feel free to copy and paste the following:

Kikuo Asai (2010). Visualization Based on Geographic Information in Augmented Reality, Augmented Reality, Soha Maad (Ed.), ISBN: 978-953-7619-69-5, InTech, Available from:

http://www.intechopen.com/books/augmented-reality/visualization-based-on-geographic-information-inaugmented-reality

\section{INTECH}

open science / open minds

\section{InTech Europe}

University Campus STeP Ri

Slavka Krautzeka 83/A

51000 Rijeka, Croatia

Phone: +385 (51) 770447

Fax: +385 (51) 686166

www.intechopen.com

\section{InTech China}

Unit 405, Office Block, Hotel Equatorial Shanghai

No.65, Yan An Road (West), Shanghai, 200040, China

中国上海市延安西路65号上海国际贵都大饭店办公楼405单元

Phone: +86-21-62489820

Fax: +86-21-62489821 
(C) 2010 The Author(s). Licensee IntechOpen. This chapter is distributed under the terms of the Creative Commons Attribution-NonCommercialShareAlike-3.0 License, which permits use, distribution and reproduction for non-commercial purposes, provided the original is properly cited and derivative works building on this content are distributed under the same license. 